\title{
Exodontia de mesiodens em palato: relato de caso
}

\author{
Extraction of mesiodens on palate: case report \\ Extracción de mesiodens en paladar: relato de caso \\ Maria de Nazaré dos SANTOS ${ }^{1}$ \\ Fabrício dos Santos DINIZ ${ }^{2}$ \\ Jéssica Vasconcelos MATTOS ${ }^{3}$ \\ José Maurício de Souza Cruz VELOSO FILHO ${ }^{4}$ \\ Rafaela Cardoso de $\mathbf{S A ́}^{\mathbf{5}}$
}
${ }^{I}$ Pós-graduação Lato Sensu em Endodontia, Universidade do Estado do Amazonas (UEA), Cirurgiã-Dentista Faculdade de Odontologia - IAES, Manaus-AM, Brasil ${ }^{2}$ Pós-Graduando Lato Sensu em Prótese Dentária na Universidade do Estado do Amazonas(UEA) Manaus-AM, Brasil ${ }^{3}$ Pós-Graduanda Lato Sensu em Ortodontia, Instituto Brasileiro do Ensino do Norte (IBEN) Manaus-AM, Brasil ${ }^{4}$ Mestrado em Implantodontia, Centro Universitário da Fundação Educacional de Barretos (UNIFEB) Barretos-SP, Brasil, Docente das Disciplinas de Prótese Parcial Removível, Prótese Total e Prótese Fixa, Anatomia Geral e Fisiologia do Instituto Amazônia de Ensino Superior (IAES) Manaus-AM, Brasil ${ }^{5}$ Mestrado profissional em Odontologia, Centro de Pesquisas Odontológicas São Leopoldo Mandic (SLMANDIC) Campinas-SP, Brasil, Docente da Disciplina de Odontopediatria do Instituto Amazônia de Ensino Superior (IAES) Manaus-AM, Brasil

\section{Resumo}

Introdução: Os dentes supranumerários são aqueles além da quantidade normal na arcada. O tipo mais comum é o mesiodens localizado entre os incisivos centrais superiores, possui geralmente forma conoide e raiz curta. Objetivo: O objetivo deste trabalho é relatar um caso clínico de exodontia de um mesiodens com abordagem cirúrgica realizada pela palatina. Caso clínico: Paciente sexo masculino, 8 anos de idade, compareceu com o responsável à clínica do IAES com queixa de dificuldades na mastigação e fala, além de vergonha de sorrir por causa de "um dente a mais". Durante o exame clínico constatou-se a presença de um elemento conoide erupcionado posteriormente aos incisivos centrais superiores. Diante dos achados clínico e radiográfico, o diagnóstico foi de mesiodens. O tratamento proposto foi a exodontia do elemento. O pós-operatório foi satisfatório, com uma boa epitelização da ferida cirúrgica, além da diminuição das dificuldades na mastigação e fala do paciente. Conclusão: A exodontia surge como um tratamento eficaz na resolução de dentes supranumerários.

Descritores: Dente Supranumerário; Extração Dentária; Cirurgia Bucal.

\section{Abstract}

Introduction: Supernumerary teeth are those beyond the normal amount in the arch. The most common type is the mesiodens located between the upper central incisors, usually having a conid and short root shape. Objective: The objective of this study is to report a clinical case of a mesiodens with a surgical approach performed by the palatine. Case report: A male patient, 8 years old, attended the IAES clinical clinic complaining of difficulties in chewing and speaking, as well as being ashamed to smile because of "one more tooth". During the clinical examination, the presence of a conid element erupted posteriorly to the upper central incisors was observed. Facing the clinical and radiographic findings, the diagnosis was mesiodens. The proposed treatment was the extraction of the element. The postoperative was satisfactory, with a good epithelization of the surgical wound, besides the reduction of the difficulties in chewing and speaking of the patient. Conclusion: Exodontia appears as an effective treatment in the resolution of supernumerary teeth.

Descriptors: Tooth, Supernumerary; Tooth Extraction, Surgery, Oral.

\section{Resumen}

Introducción: Los dientes supernumerarios son aquellos más allá de la cantidad normal en la arcada. El tipo más común es el mesiodens localizado entre los incisivos centrales superiores, generalmente tiene forma conóide y raíz corta. Objetivo: El objetivo de este trabajo es relatar un caso clínico de extracción de un mesiodens con abordaje quirúrgico realizado por la palatina. Caso clínico: Paciente sexo masculino, 8 años de edad, asistió al responsable de la clínica del IAES con queja de dificultades en la masticación y habla, además de vergüenza de sonreír a causa de un diente más. Durante el examen clínico se constató la presencia de un elemento conoide erupcionado posteriormente a los incisivos centrales superiores. Ante los hallazgos clínico y radiográfico, el diagnóstico fue de mesiodens. El tratamiento propuesto fue la exodoncia del elemento. El postoperatorio fue satisfactorio, con una buena epitelización de la herida quirúrgica, además de la disminución de las dificultades en la masticación y habla del paciente. Conclusión: La extracción surge como un tratamiento eficaz en la resolución de dientes supernumerarios.

Descriptores: Diente Supernumerario; Extracción Dental; Cirugía Bucal.

\section{INTRODUÇÃO}

Os dentes supranumerários são caracterizados por uma hiperatividade da lâmina dentária, resultando em dentes além da quantidade normal na arcada ${ }^{1}$. Podem ocorrer de forma isolada ou múltipla, unilateral ou bilateral, afetar tanto maxila como a mandíbula e ambas as dentições.

Algumas complicações associadas aos dentes supranumerários incluem impactação, erupção tardia, diastemas, erupção ectópica e formação de cistos ${ }^{2}$. Essa condição pode estar associada a algumas desordens genéticas como síndrome de Gardner, disostose cleidocraniana, fendas palatinas e fissuras labiais ${ }^{3}$.

O mesiodens representa cerca de $80 \%$ dos supranumerários e é localizado na maxila entre os incisivos centrais superiores. Possui uma forma conoide, raiz curta e na maioria dos casos encontra-se impactado ${ }^{4}$. Estudos relatam que indivíduos do sexo masculino são mais afetados na proporção $2: 1^{5-8}$.
O diagnóstico precoce, realizado por meio de exame clínico e radiográfico, é de suma importância, já que possibilita um plano de tratamento adequado e favorece o prognóstico ${ }^{9,10}$. A exodontia preventiva pode pôr em risco o desenvolvimento do germe permanente, se houver lesão acidental. Nesses casos pode ser feito acompanhamento, aguardando-se a rizogênese dos dentes adjacentes. A exodontia logo após a detecção também pode ser considerada, pois evita futuras complicações decorrente do supranumerário ${ }^{11}$. Desta forma, o objetivo deste trabalho foi relatar um caso clínico de exodontia de dente supranumerário classificado como mesiodens com a abordagem cirúrgica realizada pela região palatina.

\section{CASO CLÍNICO}

Paciente do sexo masculino, 8 anos de idade, compareceu com o responsável à clínica de 
Odontopediatria da Faculdade IAES, encaminhado para avaliação de um elemento na região do palato entre os elementos 11 e $21 . \mathrm{Na}$ anamnese o responsável relatou que paciente tinha dificuldades na mastigação e na fala, além de vergonha de sorrir na escola porque tinha "um dente a mais". Também relatou que o paciente não apresentava nenhuma outra complicação sistêmica. Durante o exame clínico observou-se que o paciente possuia dentição mista e foi verificado um elemento dentário na região da maxila, entre os incisivos centrais permanentes (Figuras 1 e 2).

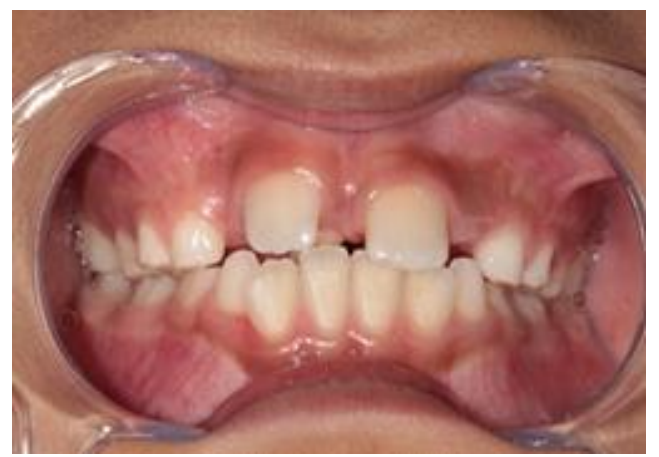

Figura 1: Mesiodens vista frontal.

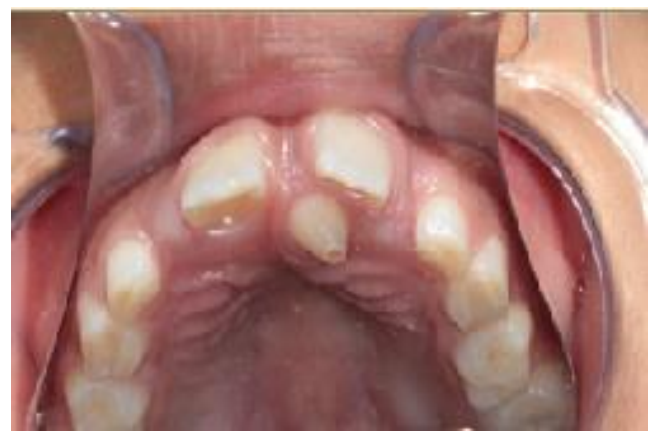

Figura 2: Mesiodens vista oclusal.

O paciente já possuía os exames complementares radiográficos: periapical (Figura 3) e panorâmica (Figura 4). Diante dos achados clínicos e radiográficos, o diagnóstico do paciente foi a presença de mesiodens com forma conoide. $\mathrm{O}$ tratamento proposto para a resolução deste caso foi a exodontia do supranumerário. Após a antissepsia intra e extraoral realizou-se a técnica anestésica utilizando Lidocaína 2\% com epinefrina 1:100.000, sendo feito o bloqueio do nervo nasopalatino e infiltrativa na região vestibular.

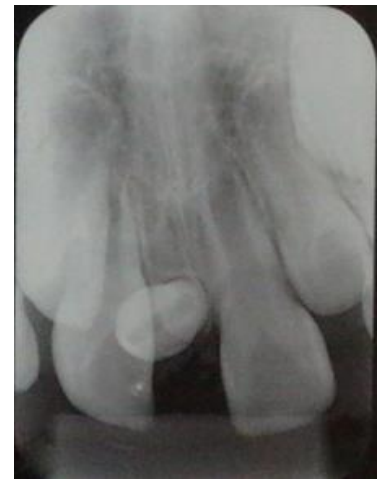

Figura 3: Aspecto radiográfico periapical

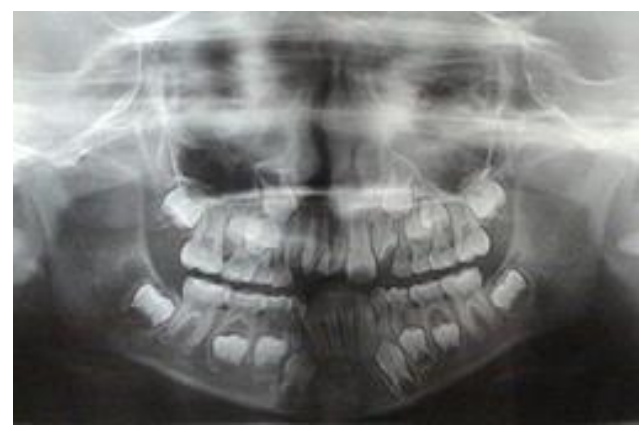

Figura 4: Aspecto radiográfico panorâmico.

Após a incisão, ao redor do elemento dental com lâmina $15 \mathrm{c}$ (Figura 5), procedeu-se o descolamento com o descolador de Molt $\mathrm{n}^{\circ} 05$ (Figura 6) e a extração do mesiodens utilizando fórceps $n^{0} 5$ (Figuras 7 e 8). Após a remoção, a loja cirúrgica foi irrigada com soro fisiológico $0,9 \%$ e suturado com fio de seda $\mathrm{n}^{\mathrm{o}} 3.0$ (Figuras 9 e 10). Após a cirurgia, foi prescrito anti-inflamatório por dois dias e as recomendações pós-operatórias. Depois de sete dias, foi realizada a remoção da sutura e acompanhamento pós-operatório (Figura 11).

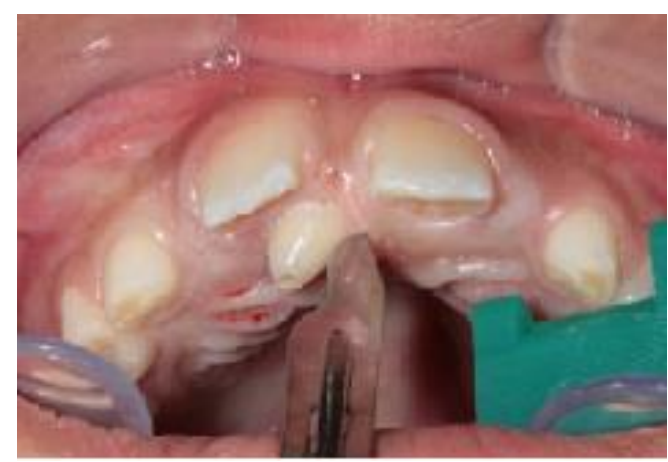

Figura 5: Incisão.

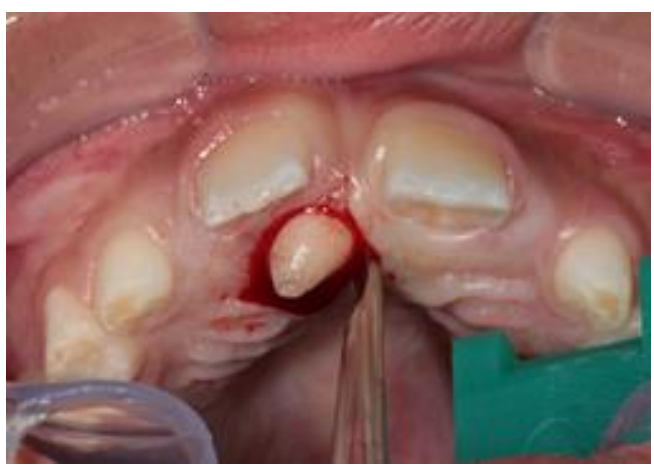

Figura 6: Sindesmotomia.

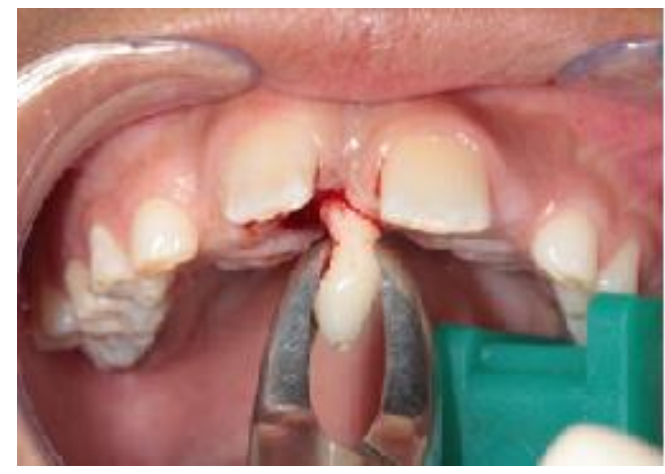

Figura 7: Exérese. 


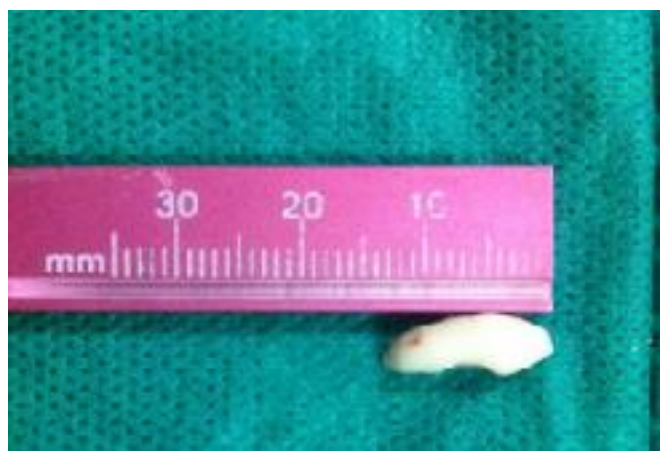

Figura 8: Dente supranumerário.

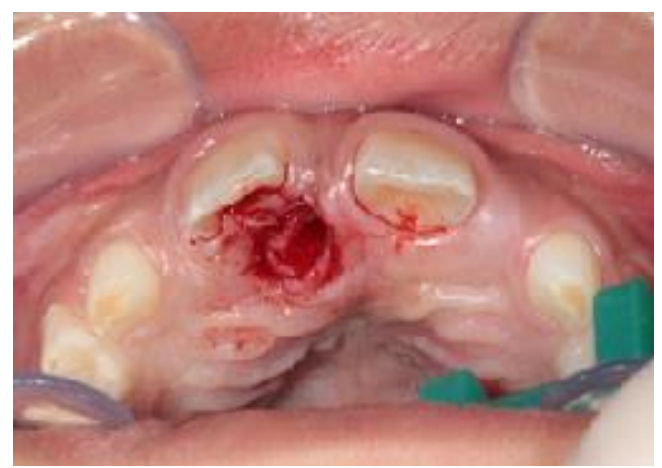

Figura 9: Aspecto pós-cirúrgico.

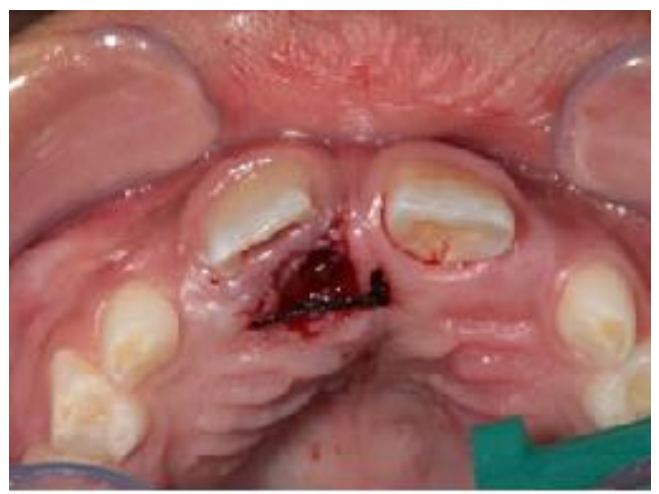

Figura 10: Sutura.

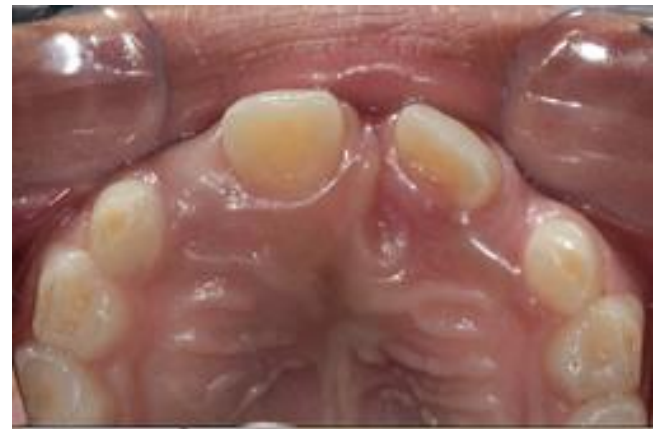

Figura 11: Aspecto clínico após 7 dias.

\section{DISCUSSÃO}

O tipo mais comum de supranumerário é o mesiodens com maior frequência no sexo masculino do que no feminino ${ }^{8}$, na faixa etária de 6-9 anos de idade. Apenas $25 \%$ dos mesiodens erupcionam ${ }^{12}$, $50,3 \%$ possuem formato cônico e cerca de $80 \%$ são localizados na região palatina, próximo aos incisivos centrais superiores $^{10}$.

No presente caso clínico, o paciente de 8 anos do sexo masculino está dentro das estatísticas citadas e, após os exames clínicos e radiográficos, verificou-se que o mesiodens apresentava-se erupcionado pela palatina e com formato cônico.

Os dentes supranumerários podem permanecer por muito tempo sem trazer qualquer dano, assim, deve-se seguir as indicações de tratamento, sendo possível apenas o acompanhamento radiográfico e clínico, ou até mesmo intervenção cirúrgica e o uso de recursos ortodônticos $^{7,13}$. Se o mesmo estiver interferindo na relação normal da oclusão, deve ser extraído, desde que não prejudique o desenvolvimento radicular dos dentes adjacentes ${ }^{11}$.

As anomalias de número dos órgãos dentários podem causar alterações na função mastigatória e fonação, assim como os problemas estéticos que podem afetar a vida social do indivíduo. A não extração do supranumerário também pode acarretar problemas inflamatórios na mucosa bucal, atraso na erupção dos permanentes, reabsorção radicular dos dentes adjacentes e edema cístico na região pré-maxilar ${ }^{11}$. Podem também ocorrer erupção ectópica, levando a mordida cruzada na região e aparecimento de diastema ${ }^{12}$. No presente caso clínico, o mesiodens interferiu na posição do elemento 21 , fazendo com que o mesmo erupcionasse mais vestibularizado.

Considerando todas as complicações apresentadas pelo paciente, tais como dificuldades na fonação e mastigação, além de diastemas, a exodontia foi o tratamento de escolha e obteve-se um resultado satisfatório, com adequada cicatrização da ferida cirúrgica e resolução das queixas iniciais.

CONCLUSÃO

A exodontia de dentes supranumerários surge como um tratamento eficaz na resolução do quadro, contribuindo para diminuição das dificuldade na mastigação e fonação. Porém é importante atentar-se para não haver lesões acidentais aos dentes adjacentes.

\section{REFERÊNCIAS}

1. Mafra RP, Vasconcelos RG, Vasconcelos MG, Queiroz LMG, Barboza CAG. Desenvolvimento dental: aspectos morfogenéticos e relações com as anomalias dentárias do desenvolvimento. Rev Bras Odontol. 2012;69(2):232-37.

2. Ata-Ali F, Ata-Ali J, Peñarrocha-Oltra D, Peñarrocha-Diago M. Prevalence, etiology, diagnosis, treatment and complications of supernumerary teeh. J Clin Exp Dent. 2014;6(4):414-18.

3. Subasioglu A, Savas S, Kucukyilmaz E, Kesim S, Yagci A, Dundar M. Genetic background of supernumerary teeth. Eur J Dent. 2015;9(1):153-58.

4. Marchetti G, Oliveira RV. Mesiodens - dentes supranumerários: diagnóstico, causas e tratamento. Rev UNINGA. 2015;24(1):19-23.

5. Mahabob MN, Anbuselvan GJ, Kumar BS, Raja S, Kothari S. Prevalence rate of supernumerary 
teeth among non-syndromic South Indian population: an analysis. J Pharm Bioall Sci. 2012;4(Suppl 2):S373-75.

6. Villavicencio J, Hernández J, Medina S. Clinical variations of double mesiodens: a review and case report. Rev Fac Odontol Univ Antioq. 2015;27(1):216-27.

7. Bereket C, Çakir-Özkan N, Şener İ, Bulut E, Baştan Aİ. Analyses of 1100 supernumerary teeth in a nonsyndromic Turkish population: a retrospective multicenter study. Niger $\mathbf{J}$ Clin Pract. 2015;18(6):731-38.

8. Burhan AS, Nawaya FR, Arabi-Katbi ME, AlJawabra AS. Prevalence of supernumerary teeth in a nonsyndromic Syrian sample. J Egypt Public Health Assoc. 2015;90(4):146-49.

9. Nunes KM, Medeiros MV, Ceretta LB, Simões PW, Azambuja FG, Sônego FGF et al. Dente supranumerário: revisão bibliográfica e relato de caso clínico. Rev Odontol Univ Cid São Paulo. 2015;27(1):72-81.

10.Nam OH, Lee HS, Kim MS, Yun KH, Bang JB, Choi SC. Characteristics of mesiodens and its related complications. Pediatr Dent. 2015;37(7):e105-9.

11. Shih WY, Hsieh CY, Tsai TP. Clinical evaluation of the timing of mesiodens removal. J Chin Med Assoc. 2016;79(6):345-50.

12.Lara TS, Lancia M, Silva Filho OGS, Garib DG, Ozawa TO. Prevalence of mesiodens in orthodontic patients with deciduous and mixed dentition and its association with other dental anomalies. Dental Press J Orthod. 2013;18(6):93-9.

13.Silva IJP, Magalhães AKC, Carneiro GKM, Favretto CO. Fechamento de diastema interincisal superior associado a dente supranumerário com intervenção ortodôntica: relato de caso clínico. Arch Health Invest. 2019;8(3):130-33.

\section{CONFLITO DE INTERESSES}

Os autores declaram não haver conflitos de interesse.

AUTOR PARA CORRESPONDENCIA

\section{Maria Nazaré dos Santos}

dranazaresantoss@gmail.com

Submetido em 22/08/2018

Aceito em 12/03/2019 